\title{
FACTORIZATION OF QUASI-DIFFERENTIAL OPERATORS
}

\author{
W. N. EVERITT, JAMES S. MULDOWNEY, AND NEEZA THANDI
}

(Communicated by Kenneth R. Meyer)

\begin{abstract}
A quasi-differential generalization of operators of the form $l_{n} u=$ $u^{(n)}+p_{1} u^{(n-1)}+\cdots+p_{n} u$ is considered. This type of generalization was first formulated by M. Bôcher (1913). A result of A. Zettl (1971) giving a necessary and sufficient condition that a differential operator $l_{n}$ be factorable into a product of lower order differential operators is extended to quasi-differential expressions.
\end{abstract}

\section{INTRODUCTION}

Let $I$ be a real interval and let $A=\left[a_{s}^{r}\right]$ be a set of complex-valued functions on $I$ such that

$$
a_{r+1}^{r} \neq 0, \quad r=0,1,2, \ldots
$$

a.e. $I$ and

$$
a_{s}^{r}, \quad s=1,2, \ldots, r+1, r=1,2, \ldots
$$

are locally Lebesgue integrable on $I$. Such a system $A$ will be called admissible in this paper. If $u$ is a complex-valued function on $I$, the quasi-derivatives $u_{A}^{[r]}, r=0,1,2, \ldots$, determined by an admissible system $A$ are defined inductively by

$$
\begin{aligned}
& a_{1}^{0} u_{A}^{[0]}=u \\
& a_{r+1}^{r} u_{A}^{[r]}=\left(u_{A}^{[r-1]}\right)^{\prime}-\sum_{j=1}^{r} a_{j}^{r} u_{A}^{[j-1]}, \quad r=1,2, \ldots
\end{aligned}
$$

whenever these expressions exist and where the prime denotes ordinary differentiation. The subscript $A$ will be suppressed so that $u_{A}^{[r]}=u^{[r]}$ if the admissible system $A$ in question is not to be emphasized.

When $a_{s}^{r}=0, s \neq r+1$, and $a_{r+1}^{r}=1, r=0,1,2, \ldots,(1.1)$ gives $u^{[r]}=u^{(r)}$, the ordinary derivative of order $r$ of $u$.

Received by the editors March 3, 1990.

1980 Mathematics Subject Classification (1985 Revision). Primary 34A30, 34C10.

The research of the second author was supported by NSERC Grant 7197.

The research of the third author was supported by an NSERC Summer Research Scholarship. 
A linear function $l_{n}$ with domain and range in the set of complex-valued functions on $I$ is a linear quasi-differential operator of order $n$ if

$$
l_{n} u=u_{A}^{[n]}+p_{1} u_{A}^{[n-1]}+\cdots+p_{n} u_{A}^{[0]},
$$

where $a_{n+1}^{n} p_{i}$ are locally Lebesgue integrable on $I, i=1, \ldots, n$. The theory of linear ordinary differential equations extends in a natural way to quasidifferential equations of the form

$$
l_{n} u=f,
$$

where $a_{n+1}^{n} f$ is locally Lebesgue integrable on $I$. A solution of (1.3) is any function $u$ such that $u^{[s]}, s=0, \ldots, n-1$ are locally absolutely continuous on $I$ and (1.3) holds almost everywhere on $I$. For discussions of existence and uniqueness results, spectral and algebraic properties, and control theory for quasi-differential equations, see Zettl [13], Everitt [5], and the references there.

It should be noted that the definition of a quasi-differential operator of order $n$ given here differs from that given by Zettl in [13]. There, in our notation, $a_{1}^{0}=a_{n+1}^{n}=1$ in (1.1) and $p_{1}=\cdots=p_{n}=0$ in (1.2). In fact, no greater generality is achieved by us in relaxing these conditions and the two concepts are equivalent to within changes of variable. However it is notationally convenient for the formulation and proof of our results to present the operators in this form.

The first consideration in generality of this concept seems to be due to Bôcher [1] who also used the term quasi-differential equation, [2, p. 36]. Zettl ([13, p. 455]) discusses the advantages of quasi-differential operators which include not only their greater generality but also a simplicity that they bring to the formulation of adjoint problems.

Here we are interested in extending to quasi-differential operators of the form (1.2) a result proved by Zettl [12] for ordinary differential operators. This result states that a linear ordinary differential operator $l_{n}$ of order $n$ may be factored in the form $l_{n}=m_{n-p} l_{p}$, where $l_{p}, m_{n-p}$ are linear differential operators of order $p, n-p$ respectively, if and only if the equation $l_{n} u=0$ has solutions $u_{1}, \ldots, u_{p}$ such that $W\left(u_{1}, \ldots, u_{p}\right) \neq 0$ on $I$, where $W$ is the Wronskian determinant. In [8, Corollary 3.2] it is shown that this follows from Sylvester's Identity for determinants. This approach also works in the present context. The main difficulty is that the resulting entity $m_{n-p}$ is not necessarily a quasidifferential operator with respect to the original system $A$. However we will show that there is an admissible system $B$ with respect to which $m_{n-p}$ is a quasi-differential operator of order $n-p$.

A brief note on the early history of factorization of differential operators is given by Hartman at the end of Chapter IV of [7], where the subject is explored in the context of reduction of order in systems where some solutions are known. Also see Pólya [11] and Coppel [3, p. 138] where the connection between what is now called disconjugacy and the factorization of a linear differential operator into a product of first order operators is discussed. This discussion is extended 
by Hartman, [7, Chapter IV, Corollary 3.1] to quasi-differential operators although that terminology is not used. This result is also obtained by Nehari [10] for a somewhat more restricted class of quasi-differential operators. The relationship between factorization and disconjugacy is further developed in [4] where many useful references are given.

\section{A FACTORIZATION THEOREM}

Let $A$ be an admissible system of functions on $I$ as described in $\S 1$. Denote by $D_{n}(A)$ the set of complex-valued functions $u$ on $I$ such that $u_{A}^{[0]}, \ldots, u_{A}^{[n-1]}$ exist and are locally absolutely continuous on $I$. From the discussions in [5, 13], it is clear that $D_{n}(A)$ is an infinite-dimensional linear space. Let $L_{n}(A)$ denote the set of quasi-differential operators $l_{n}$ of order $n$ as described in (1.2).

For any matrix $\left[w_{s}^{r}\right]$, let $w_{s_{1} \cdots s_{p}}^{r_{1} \cdots r_{p}}$ denote the minor determined by the rows $r_{1}, \ldots, r_{p}$ and the columns $s_{1}, \ldots, s_{p}$. In particular, when $l_{n} \in L_{n}(A)$ and $u_{1}, \ldots, u_{p}$ are solutions of $l_{n} u=0$, we define the quasi-Wronskian as the determinant $W_{A}\left(u_{1}, \ldots, u_{p}\right)=w_{12 \cdots p}^{12 \cdots p}$, where $w_{s}^{r}=u_{s}^{[r-1]}, r, s=1,2, \ldots, p$.

If $A, B$ are both admissible, we say that $l_{n} \in L_{n}(A)$ may be factored in the form $l_{n}=m_{n-p} l_{p}$, where $l_{p} \in L_{p}(A)$ and $m_{n-p} \in L_{n-p}(B)$, when $l_{p} u \in$ $D_{n-p}(B)$ for each $u \in D_{n}(A)$ and $m_{n-p} l_{p} u=l_{n} u$ for each such $u$. We prove the following result.

Theorem 2.1. Let $l_{n} \in L_{n}(A)$. Then $l_{n}$ may be factored in the form $l_{n}=$ $m_{n-p} l_{p}$, where $l_{p} \in L_{p}(A)$ and $m_{n-p} \in L_{n-p}(B)$ for some admissible system $B$ if and only if there exist solutions $u_{1}, \ldots, u_{p}$ of $l_{n} u=0$ such that

$$
W_{A}\left(u_{1}, \ldots, u_{p}\right) \neq 0 \text { on } I \text {. }
$$

Successive applications of this theorem with $p=n-1, n-2, \ldots$ yield the following result of Hartman [7, Chapter IV, Corollary 3.1] which was proved originally for ordinary differential operators by Pólya [11].

Corollary 2.2. A necessary and sufficient condition so that $l_{n} \in L_{n}(A)$ can be expressed as a product of $n$ first order quasi-differential operators is that $l_{n} u=$ 0 have solutions $u_{1}, \ldots, u_{n-1}$ such that $W_{A}\left(u_{1}, \ldots, u_{p}\right) \neq 0$ on $I, p=$ $1, \ldots, n-1$.

First observe that, as in the case of ordinary differential equations, solutions $u_{1}, \ldots, u_{n}$ of $l_{n} u=0$ form a basis for the solution space if and only if $W_{A}\left(u_{1}, \ldots, u_{n}\right) \neq 0$ on $I$. Furthermore, for such a basis,

$$
l_{n} u=W_{A}\left(u_{1}, \ldots, u_{n}, u\right) / W_{A}\left(u_{1}, \ldots, u_{n}\right)
$$

for all $u \in D_{n}(A)$ since both sides of $(2.1)$ are operators in $L_{n}(A)$ and the condition $W_{A}\left(u_{1}, \ldots, u_{n}\right) \neq 0$ implies the coefficients of $u^{[r]}, r=0, \ldots, n-1$, are the same for both operators. The necessity of the condition $W_{A}\left(u_{1}, \ldots, u_{p}\right) \neq 0$ for the factorization $l_{n}=m_{n-p} l_{p}$ is thus obvious since solutions $u_{1}, \ldots, u_{p}$ of $l_{p} u=0$ must also be solutions of $l_{n} u=0$. 
To prove the sufficiency of the condition for factorization, the following two determinant identities which hold for any $n \times n$ matrix $\left[w_{s}^{r}\right]$ are needed. If $v_{s}^{r}=w_{12 \cdots p, p+s}^{12 \cdots p, p+r}$, then

$$
w_{12 \cdots n}^{12 \cdots n}\left(w_{12 \cdots p}^{12 \cdots p}\right)^{n-p-1}=v_{12 \cdots n-p}^{12 \cdots n-p} .
$$

If $(1, \ldots, \hat{j}, \ldots, n)$ denotes the $(n-1)$-tuple obtained by omitting $j$ from $(1, \ldots, n)$ and $w(i, x)_{s_{1} \cdots s_{m}}^{r_{1} \cdots r_{m}}$ is the minor determined by the rows $r_{1}, \ldots, r_{m}$ and the columns $s_{1}, \ldots, s_{m}$ of $\left[w_{s}^{r}\right]$ with the $i$ th row $\left[w_{1}^{i}, \ldots, w_{n}^{i}\right]$ replaced by $x=\left[x_{1}, \ldots, x_{n}\right]$, then if $1 \leq i, j \leq n$ and $i \neq j$,

$$
\begin{aligned}
w(i, x)_{1 \cdots n-1}^{1 \cdots \hat{j} \cdots n} w_{12 \cdots n}^{12 \cdots n}= & w_{1 \cdots n-1}^{1 \cdots \hat{j} \cdots n} w(i, x)_{12 \cdots n}^{12 \cdots n} \\
& +(-1)^{i-j+1} w_{1 \cdots n-1}^{1 \cdots \hat{i} \cdots n} w(j, x)_{12 \cdots n}^{12 \cdots n} .
\end{aligned}
$$

The expression (2.2) is Sylvester's Identity (see [6, p. 32]) and a very simple proof of $(2.3)$ may be found in [9, p. 65].

Now suppose $u_{1}, \ldots, u_{p}$ are as specified with $W_{A}\left(u_{1}, \ldots, u_{p}\right) \neq 0$ and choose $u_{p+1}, \ldots, u_{n}$ such that $W_{A}\left(u_{1}, \ldots, u_{n}\right) \neq 0$ so $u_{1}, \ldots, u_{n}$ is a basis for the solution set of $l_{n} u=0$. Consider the $(n+1) \times(n+1)$ matrix $\left[w_{s}^{r}\right]$ defined by $w_{s}^{r}=u_{s}^{[r-1]}, r, s=1, \ldots, n+1$ and let $u_{n+1}=u$, an arbitrary function in $D_{n}(A)$. Now, from (2.1), $l_{n} u=w_{12 \cdots n+1}^{12 \cdots n+1} / w_{12 \cdots n}^{12 \cdots n}$ and, applying Sylvester's Identity (2.2) to both numerator and denominator here, we find

$$
l_{n} u=v_{12 \cdots n-p+1}^{12 \cdots n-p+1} / v_{12 \cdots n-p}^{12 \cdots n-p} w_{12 \cdots p}^{12 \cdots p},
$$

where $v_{s}^{r}=w_{12 \cdots p, p+s}^{12 \cdots p, p+r}$. To complete the proof, we must show that (2.4) may be written in the form

$$
l_{n} u=m_{n-p} v, \quad v=l_{p} u=w_{12 \cdots p, n+1}^{12 \cdots p, p+1} / w_{12 \cdots p}^{12 \cdots p},
$$

where $m_{n-p} \in L_{n-p}(B)$ for some admissible system $B=\left[b_{s}^{r}\right]$. To see this, consider

$$
\begin{aligned}
& v_{s}^{\{r-1\}}=v_{s}^{r}=w_{12 \cdots p, p+s}^{12 \cdots p, p}, \quad r=1, \ldots, n-p, \\
& v_{s}^{\{n-p\}}=v_{s}^{n-p+1} / w_{12 \cdots p}^{12 \cdots p}=w_{12 \cdots p, p+s}^{12 \cdots p, n+1} / w_{12 \cdots p}^{12 \cdots p},
\end{aligned}
$$

for $s=1, \ldots, n+1-p$. We will find an admissible system $B$ such that

$$
\begin{aligned}
& b_{1}^{0} v_{s}^{\{0\}}=v_{s}=l_{p} u_{p+s} \\
& b_{r+1}^{r} v_{s}^{\{r\}}=\left(v_{s}^{\{r-1\}}\right)^{\prime}-\sum_{j=1}^{r} b_{j}^{r} v_{s}^{\{j-1\}}, \quad r=1, \ldots, n-p .
\end{aligned}
$$

This shows that $v_{s}^{\{r\}}=\left(v_{s}\right)_{B}^{[r]}, s=1, \ldots, n+1-p, r=0, \ldots, n-p$; and (2.4), (2.6), (2.7) establish (2.5) with

$$
m_{n-p} v=W_{B}\left(l_{p} u_{p+1}, \ldots, l_{p} u_{n}, v\right) / W_{B}\left(l_{p} u_{p+1}, \ldots, l_{p} u_{n}\right) .
$$


We now find the system $B$ satisfying (2.7). The first line of (2.7) is satisfied if $b_{1}^{0}=1 / w_{12 \cdots p}^{12 \cdots p}$. Next, from (1.1), with $b_{r+1}^{r}=a_{p+r+1}^{p+r}, r=1, \ldots, n-p-1$, and

$$
\begin{aligned}
& b_{n-p+1}^{n-p}=a_{n+1}^{n} w_{12 \cdots p}^{12 \cdots p}, \\
& b_{r+1}^{r} v_{s}^{\{r\}}=w(p+1, x)_{12 \cdots p, p+s}^{12 \cdots p, p+1}-\sum_{j=1}^{r} a_{p+j}^{p+r} v_{s}^{\{j-1\}}, \quad r=1, \ldots, n-p,
\end{aligned}
$$

where $x=\left[\left(u_{1}^{[p+r-1]}\right)^{\prime}, \ldots,\left(u_{p}^{[p+r-1]}\right)^{\prime},\left(u_{p+s}^{[p+r-1]}\right)^{\prime}\right]$. Also, from the rule for differentiating determinants,

$$
\left(v_{s}^{\{r-1\}}\right)^{\prime}=w(p+1, x)_{12 \cdots p, p+s}^{12 \cdots p, p+1}+w(p, y)_{12 \cdots p, p+s}^{12 \cdots p, p+r}, \quad r=1, \ldots, n-p,
$$

where $y=\left[\left(u_{1}^{[p-1]}\right)^{\prime}, \ldots,\left(u_{p}^{[p-1]}\right)^{\prime},\left(u_{p+s}^{[p-1]}\right)^{\prime}\right]$. Combining (2.8) and (2.9), we find

$$
b_{r+1}^{r} v_{s}^{\{r\}}=\left(v_{s}^{\{r-1\}}\right)^{\prime}-\sum_{j=1}^{r} a_{p+j}^{p+r} v_{s}^{\{j-1\}}-w(p, y)_{12 \cdots p, p+s}^{12 \cdots p, p+r} .
$$

Now, from (2.3), with $n=p+1, i=p, j=p+1$

$$
\begin{aligned}
w(p, y)_{12 \cdots p}^{12 \cdots p} w_{12 \cdots p, p+s}^{12 \cdots p, p+r}= & w_{12 \cdots p}^{12 \cdots p} w(p, y)_{12 \cdots p, p+s}^{12 \cdots p, p+r} \\
& +w_{12 \cdots p}^{1 \cdots p-1, p+r} w(p+1, y)_{12 \cdots p, p+s}^{12 \cdots p, p+1}
\end{aligned}
$$

which gives

$$
w_{12 \cdots p}^{12 \cdots p} w(p, y)_{12 \cdots p, p+s}^{12 \cdots p, p+r}=\left(w_{12 \cdots p}^{12 \cdots p}\right)^{\prime} v_{s}^{\{r-1\}}-w_{12 \cdots p}^{1 \cdots p-1, p+r} v_{s}^{\{0\}},
$$

since $v_{s}^{\{r-1\}}=w_{12 \cdots p, p+s}^{12 \cdots p, p+r}, v_{s}^{\{0\}}=w_{12 \cdots p, p+s}^{12 \cdots p, p+1}=w(p+1, y)_{12 \cdots p, p+s}^{12 \cdots p, 1}$, and $w(p, y)_{12 \cdots p}^{12 \cdots p}=\left(w_{12 \cdots p}^{12 \cdots p}\right)^{\prime}$. Now $(2.10),(2.11)$ show that $(2.7)$ is satisfied if $B$ is given by

$$
\begin{aligned}
& b_{1}^{0}=1 / w_{12 \cdots p}^{12 \cdots p}, b_{n-p+1}^{n-p}=a_{n+1}^{n} w_{12 \cdots p}^{12 \cdots p} \\
& b_{r+1}^{r}=a_{p+r+1}^{p+r}, r=1, \ldots, n-p-1, \\
& b_{s}^{r}=a_{p+r}^{p+r}, s=2, \ldots, r-1, r=1, \ldots, n-p \\
& b_{1}^{r}=a_{p+r}^{p+r}-w_{12 \cdots p}^{1 \cdots p-1, p+r} / w_{12 \cdots p}^{12 \cdots p}, \\
& b_{r}^{r}=a_{p+r}^{p+r}+\left(w_{12 \cdots p}^{12 \cdots p}\right)^{\prime} / w_{12 \cdots p}^{12 \cdots p} .
\end{aligned}
$$

The admissibility of $B$ follows from the admissibility of $A$ and $w_{12 \cdots p}^{12 \cdots p}=$ $W_{A}\left(u_{1}, \ldots, u_{p}\right) \neq 0$.

\section{REFERENCES}

1. M. Bôcher, Applications and generalizations of the concept of adjoint systems, Trans. Amer. Math. Soc. 14 (1913), 403-420.

2. __ Leçons sur les méthodes de Sturm dans la théorie des equations differentielles linéaires et leurs dévelloppements modernes, Gauthier-Villars, Paris, 1917. 
3. W. A. Coppel, Disconjugacy, Lecture Notes in Math., vol. 20, Springer-Verlag, New York, 1971.

4. G. J. Etgen, G. D. Jones, and W. E. Taylor, Jr., On the factorizations of ordinary linear differential operators, Trans. Amer. Math. Soc. 297 (1986), 717-728.

5. W. N. Everitt, Linear control theory and quasi-differential equations, J. Applied Math. Phys. 38 (1987), 193-203.

6. F. R. Gantmacher, The theory of matrices, vol. I, Chelsea, New York, 1960.

7. P. Hartman, Ordinary differential equations, Wiley, New York, 1964.

8. J. S. Muldowney, On an inequality of Caplygin and Pólya, Proc. Roy. Irish Acad. Sect. A 76 (1976), 85-99.

9. _ Markov systems of vector-valued functions and disconjugacy, J. Approx. Theory $\mathbf{5 9}$ (1989), 53-71.

10. Z. Nehari, Disconjugate linear differential operators, Trans. Amer. Math. Soc. 129 (1967), 500-516.

11. G. Pólya, On the mean-value theorem corresponding to a given linear homogeneous differential equation, Trans. Amer. Math. Soc. 29 (1922), 312-324.

12. A. Zettl, Factorization of differential operators, Proc. Amer. Math. Soc. 27 (1971), 425-426.

13. __ Formally self-adjoint quasi-differential operators, Rocky Mountain J. Math. 5 (1975), 453-474.

Department of Mathematics, The University of Birmingham, B152TT, Great Britain

Department of Mathematics, University of Alberta, Edmonton T6G 2G1, Canada

Department of Mathematics, University of Wisconsin, Madison, Wisconsin 53706 